\title{
Dynamics of ion solvation in a Stockmayer fluid
}

\author{
Lalith Perera and Max L. Berkowitz \\ Department of Chemistry, University of North Carolina, Chapel Hill, North Carolina, 27599
}

(Received 4 October 1991; accepted 12 November 1991)

\begin{abstract}
Molecular dynamics computer simulations were performed to study the dynamics of the ionic solvation in a Stockmayer fluid. The simulations show that the solvent relaxation proceeds in two time regimes. Most of the relaxation occurs in a short time period during which the relaxation process can be described by a Gaussian function. The long time regime can be described by an exponential relaxation. The decay exponent of the relaxation function in this regime is the same as the exponent describing the decay of the single dipole correlation function. In addition, the contribution of the rotational and translational modes of the solvent to the energy relaxation was investigated. It was found that when the rotational mode is the dominant mode of the solvent motion the relaxation occurs from the outside-in, in accordance with the Onsager "snowball" picture. When the influence of the translational mode is increased the Onsager picture breaks down.
\end{abstract}

\section{INTRODUCTION}

The dynamic response of the solvent to the sudden change of the charge distribution in a solute molecule has been the subject of many investigations, theoretical and experimental. ${ }^{1}$ While the experimental data are obtained from the applications of fast laser pulse techniques to rather complicated systems, ${ }^{2,3}$ the majority of analytical theories use rather simplified models to describe the solutions. ${ }^{4-10}$ At the same time, the computer simulations are performed on models that use a simple description of the solute and a rather realistic description of the solvent. ${ }^{11-15}$ Thus, recent simulations were performed to study the response in solvents such as water, ${ }^{11,12}$ methanol, ${ }^{13}$ and acetonitrile. ${ }^{14}$ To compare the results of the simulations with the analytical type theories, it is desirable to perform a simulation, where both solute and solvent are described by simple potentials. Therefore, in this paper we present results from such a simulation and compare them with the results from the existing analytical theories, such as the dynamical mean-spherical approximation (MSA) ${ }^{6-8}$ and theories based on the SmoluchowskiVlasov equation (SVE).$^{8-10}$ We also address the issue of the validity of Onsager's hypothesis, ${ }^{16}$ and the role of the translational and rotational motions in the relaxation. Finally, we express the hope, that simulations performed on simple systems can serve as a reference point for the development of more sophisticated molecular theories, such as the one given in Ref. 17.

\section{MODEL AND METHODOLOGY}

Our system consists of one solute particle dissolved in a Stockmayer solvent. ${ }^{18}$ The choice of this particular model is primarily due to the fact that analytical theories based on the dynamical extension of the MSA model ${ }^{6-8}$ and on the $\mathrm{SVE}^{8-10}$ are available for a model very similar to ours (the theories are done for hard spheres with embedded dipoles). In addition, the detailed knowledge of the dielectric properties of this solvent is available from molecular dynamics simulations. ${ }^{19-21}$

The interaction energy for a pair of Stockmayer parti- cles is given by the following expression:

$$
U_{i j}(r)=4 \epsilon\left[(\sigma / r)^{12}-(\sigma / r)^{6}\right]+\mu_{i} \cdot \mathbf{T}_{i j} \cdot \mu_{j},
$$

where $r$ is the distance between particles $i$ and $j, \mu_{i}$ is the dipole moment of particle $i, \mu_{j}$ is the dipole moment of particle $j, \epsilon$ and $\sigma$ are the Lennard-Jones parameters. The dipole tensor $\mathbf{T}_{i j}$ has its usual form,

$$
\mathbf{T}_{i j}=1 / r_{i j}^{3}\left[\mathbf{I}-3 \mathbf{r}_{i j} \mathbf{r}_{i j} / r_{i j}^{2}\right],
$$

where $I$ is the unit tensor.

The solute-solvent interaction is represented by the term

$$
U_{j}\left(r, \mu_{j}\right)=4 \epsilon\left[(\sigma / r)^{12}-(\sigma / r)^{6}\right]-\mu_{j} \cdot \mathbf{E},
$$

where $\mathbf{E}$ is the electric field due to the solute charge, and is given by an equation $\mathbf{E}=q \mathbf{r}_{i j} / r_{i j}^{3}$. For the neutral solute, this quantity becomes zero and the last term in Eq. (3) vanishes. The equations of motion for our system are obtained from the Lagrangian

$$
\begin{aligned}
L= & \sum_{i} \frac{1}{2} m v_{i}^{2}+\frac{1}{\mu^{2}} \sum_{i} \frac{1}{2} I \mu_{i}^{2}-\frac{1}{2} \sum_{i \neq j} \sum_{j} \boldsymbol{\mu}_{i} \cdot \mathbf{T}_{i j} \cdot \boldsymbol{\mu}_{j} \\
& -\frac{1}{2} \sum_{i} \sum_{j} u_{i j}^{\mathrm{LJ}}+\sum_{i} \lambda_{i}\left(\mu_{i}^{2}-\mu^{2}\right)+\sum_{i} \boldsymbol{\mu}_{i} \cdot \mathbf{E},
\end{aligned}
$$

where the first two terms represent translational and rotational kinetic energies of the solvent molecules, the third term represents the dipole-dipole interaction energy of the solvent, the fourth term is representing the Lennard-Jones interaction energy of the system. To preserve the magnitude of the dipole moment of the solvent we use the method of constraints. ${ }^{22}$ Therefore, the fifth term of the Lagrangian contains a constraint variable with the corresponding Lagrange multiplier. The last term in the Lagrangian is due to the solute-solvent electrostatic interaction. The solvent is characterized (in reduced units) by a dipole moment $\left(\mu^{*}\right)^{2}=3.0$, a moment of inertia $I^{*}=0.025$, a density $\rho^{*}=0.822$, and a temperature $T^{*}=1.15$. The LennardJones parameters for solute-solvent interaction were taken to be the same as for solvent-solvent interaction. The simulations were carried out in a cubic box, which contained 511 
solvent molecules and 1 solute molecule. For simplification of the treatment, the solute particle was considered to be immobile. Equations of motion for solvent molecules were integrated using the leapfrog algorithm and the rotational part of the motion was handled by the methods of constraints. A good description on the implementation of this method is found in Ref. 23. The step size in reduced units of time was chosen to be equal to 0.0025 . To facilitate the comparison of our results with the results from other simulations note that this time step is equal to $5.4 \mathrm{fs}$ if the argon parameters ( $\epsilon=119.8 \mathrm{~K}$ and $\sigma=3.405 \AA$ ) are chosen for solvent particles. Periodic boundary conditions along with the spherical cut-off convention were implemented in the calculations and the reaction field technique was used to account for the long range dipole-dipole interactions. In the reaction field calculations we used the value of the bulk dielectric constant of the Stockmayer fluid, which is 66.1 for the values of the reduced parameters given above. ${ }^{20}$

In the present work, we carried out three equilibrium simulations and three sets of nonequilibrium simulations. The first equilibrium simulation (ESI) was performed for the system with the uncharged solute, while the solute was charged $(q=1 e)$ in the second simulation (ESII). In the first two simulations the reduced moment of inertia $I^{*}$ of the solvent molecule was assigned the value of 0.025 , while in the third equilibrium simulation the value of $I^{*}$ was increased. By increasing the moment of inertia one achieves slow rotational motion of the solvent and thus amplifies the effects of translational motion on the overall dynamics. Since we do not intend to mimic the behavior of any real solvent system, we can make arbitrary changes in some parameters to gain more physical insight into the processes we discuss here. The third equilibrium simulation (ESIII) was performed using mostly the same set of parameters as used in ESI, only $I^{*}$ was increased 100 times. In all the equilibrium simulations the trajectories consisted of 20 ps equilibration period followed by $100 \mathrm{ps}$ production run. The average reduced temperature of each trajectory was maintained at $T^{*}=1.15$ by occasional rescaling of the velocities.

To mimic the solvation phenomena we use the nonequilibrium simulations technique. In these simulations the solute charge distribution is instantaneously changed and the response of the solute is measured. To quantify the solvation dynamics one calculates in the simulation the normalized response function or relaxation function

$$
S(t)=\frac{\delta E(t)-\delta E(\infty)}{\delta E(0)-\delta E(\infty)} .
$$

In Eq. (5) $\delta E(t)$ is the fluctuation in the solvent-solute interaction energy at a given time $t$, averaged over all the trajectories in a particular set of simulations. The equilibrium trajectories of the system with the neutral solute are used to generate initial configurations for the nonequilibrium trajectories. Therefore, we have selected 50 configurations (separated by $2 \mathrm{ps}$ each) from the equilibrium simulation SEI and at $t=0$ we placed a charge of $+1 e$ on the solute. To compare the results of our simulations with some analytical theories, such as MSA, in which the relaxational mechanism is due to the rotational motion only, we froze the transla- tional motion of the molecules in the first set of 50 trajectories. This set of 50 nonequilibrium trajectories we call NESI (nonequilibrium set I). To freeze the translations we imposed very heavy masses on the solvent molecules $\left(10^{10}\right.$ times the actual mass). Also, the initial linear velocities were set equal to zero to ensure that particles do not move initially. After the instantaneous charge jump, the trajectories from the molecular dynamics were monitored for 5.4 ps. (The equilibration has been achieved within $2.0 \mathrm{ps}$.)

It has been emphasized that translational motion of the solvent molecules plays a substantial role in the overall mechanism of the relaxation. This was confirmed recently by Bagchi and Chandra, ${ }^{10}$ who extended the SVE treatment to include the effects of the translational motion. They demonstrated that if translational diffusion is considerably large, the Onsager picture of the solvent relaxation occurring in a "snowball" fashion is no longer acceptable. ${ }^{24} \mathrm{We}$ tried to address this issue in two other sets of nonequilibrium simulations. In one set of simulations (NESII), consisting again of 50 trajectories, we started the trajectories from the same initial positions as in set NESI. The initial velocities of the solvent molecules, which were set equal to zero in the NESI, were now assigned from the equilibrium trajectories. The lengths of the trajectories were restricted to $2.7 \mathrm{ps}$ since the equilibration was attained within 2 ps.

The third set of nonequilibrium trajectories (NESIII) was started from 50 points of ESIII trajectory, where the moment of inertia of the solvent was 100 times larger than that used in ESI. Trajectories were continued for $22 \mathrm{ps}$ after the initial charge jump. No attempt has been made to control the temperature of the system in any of the above mentioned nonequilibrium trajectories.

\section{RESULTS AND DISCUSSION}

Using the data from equilibrium simulations, we first consider the structural properties of solvent around the solute molecule. The solute-solvent radial distribution functions (rdf's) obtained from ESI and ESII are shown in Fig. 1. The curves on this figure demonstrate the existence of the well defined solvation shell structures around the solute, regardless of the charge on the solute. In Table I we summarize the information extracted from the rdf's along with the other relevant data. As the rdf's in Fig. 1 indicate we distinguish three solvation shells around the solute. The rest of the solvent is "bulklike." The boundaries of the shells are given by the locations of the minima of the rdfs. The properties shown in Table $I$ are the averages over the shells defined by such boundaries. The orientational distribution of the angle between the solvent dipole and a vector connecting the center of the solute and the center of a solvent is shown in Fig. 2.

From Figs. 1 and 2 and Table I we can see that the incorporation of the charge on the solute site results in the increase of the sharpness of the first peak of the rdf, while the peak position shifts inward. The size of the first shell decreases and as a result the number of nearest neighbors decreases. The same is true for the second shell. At the same time, note, that the density of solvent in the first shell around the ion is larger than the density of the solvent in the first 


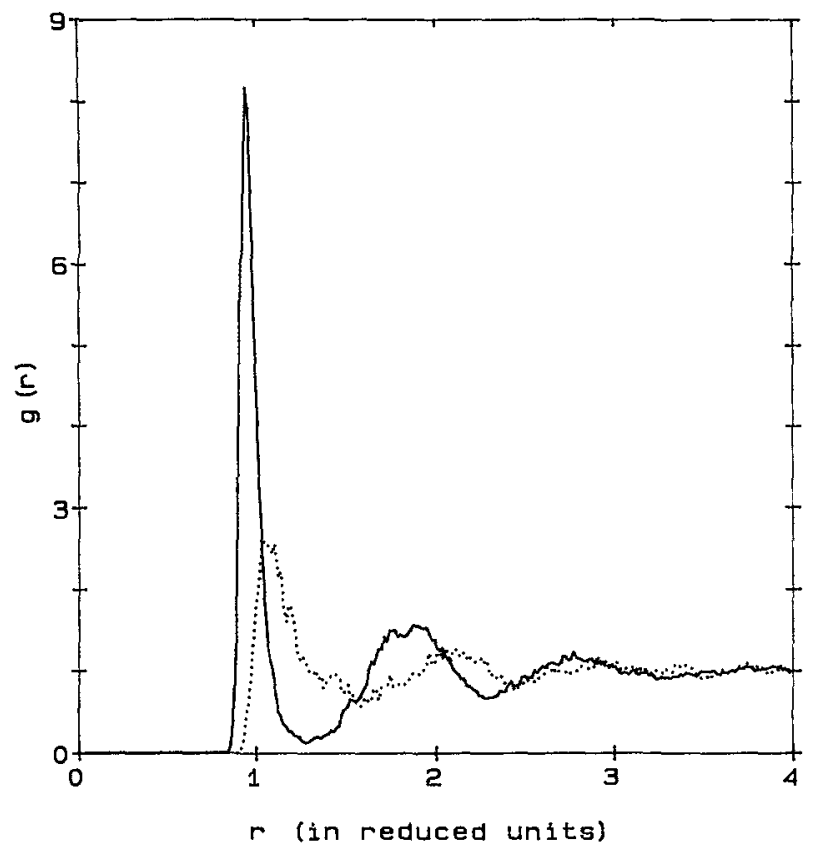

FIG. 1. The solute-solvent radial distribution functions obtained from ESI and ESII. The solid line corresponds to the ion-solvent rdf, the dotted line to the neutral solute-solvent rdf.

shell around the neutral solute. The orientational distribution is homogeneous in the case of solvated neutral solute but displays a strong orientational preference in the case when the solute is charged. As we can see in this case the orientational preference holds for all three shells around the solute. The rdf's and orientational distribution functions given by Figs. 1 and 2 represent the initial and final states of our nonequilibrium simulations.

As we have already mentioned, the response of the solvent to the instantaneous change of the solute charge distribution is measured in a molecular dynamics computer experiment by the normalized response function [see Eq. (5)].

In Fig. 3 we display the results for the correlation function $S(t)$ obtained from the NESI simulation along with the results obtained from dynamical MSA theory ${ }^{7}$ and SVE-

TABLE I. A summary of information on solvation shells. The values in the table are obtained from ESI and ESII simulations. (a) From an equilibrium simulation with a neutral solute. (b) From an equilibrium simulation with a charged solute.

\begin{tabular}{lcccc}
\hline \hline Shell & $\begin{array}{c}\text { Radius in } \\
\text { reduced units }\end{array}$ & $\begin{array}{c}\text { Number of } \\
\text { molecules }\end{array}$ & $\langle\cos (\theta)\rangle$ & $\begin{array}{c}\langle\text { Density in } \\
\text { reduced units }\end{array}$ \\
\hline 1 & 1.52 & 11.8 & -0.0061 & 0.798 \\
2 & 2.52 & 41.7 & -0.0036 & 0.800 \\
3 & 3.40 & 80.6 & -0.0004 & 0.825 \\
bulklike & & 376.9 & 0.0003 & 0.822 \\
& Radius in & Number of & & $\langle$ Density in \\
Solvation shell reduced units & molecules & $\langle\cos (\theta)\rangle$ & reduced units \\
\hline 1 & 1.27 & 9.5 & 0.889 & 1.226 \\
2 & 2.27 & 33.3 & 0.336 & 0.824 \\
3 & 3.24 & 76.4 & 0.170 & 0.817 \\
bulklike & & 391.8 & 0.054 & 0.816 \\
& & & & \\
\hline
\end{tabular}
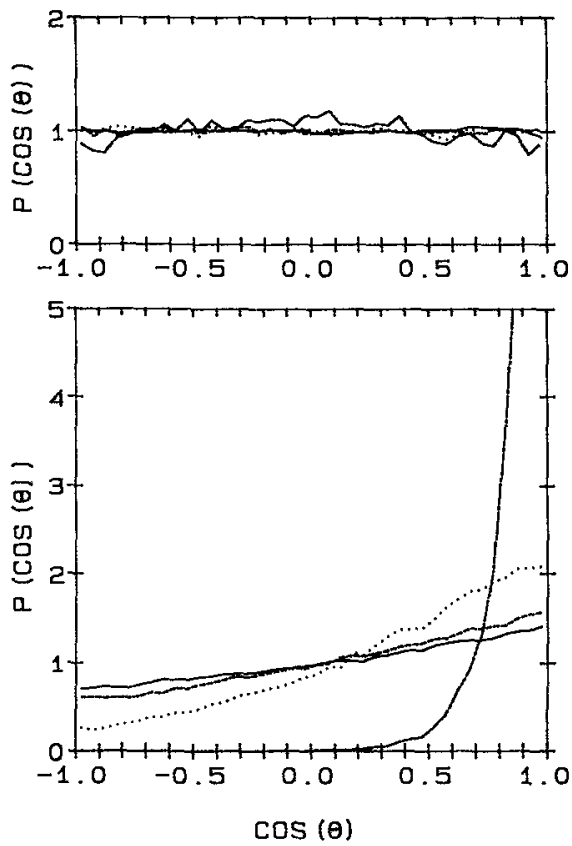

FIG. 2. Orientational distributions for different solvation shells from ESI (upper panel) and ESII (lower panel); first shell $(\cdot \cdot)$, second shell $(\cdots)$, third shell $(--)$, and bulklike $(-)$.

type theory. ${ }^{10}$ The functional forms of the relaxation function $S(t)$ obtained in the framework of MSA and SVE and used here are presented in the Appendix. Since MSA theory does not take into account the translational mode of the relaxation, the comparison of this theory with the data from NESI simulation is most appropriate. The SVER curve was obtained by solving SVE where only the rotational mode of the relaxation was included. Finally, to complete the picture,

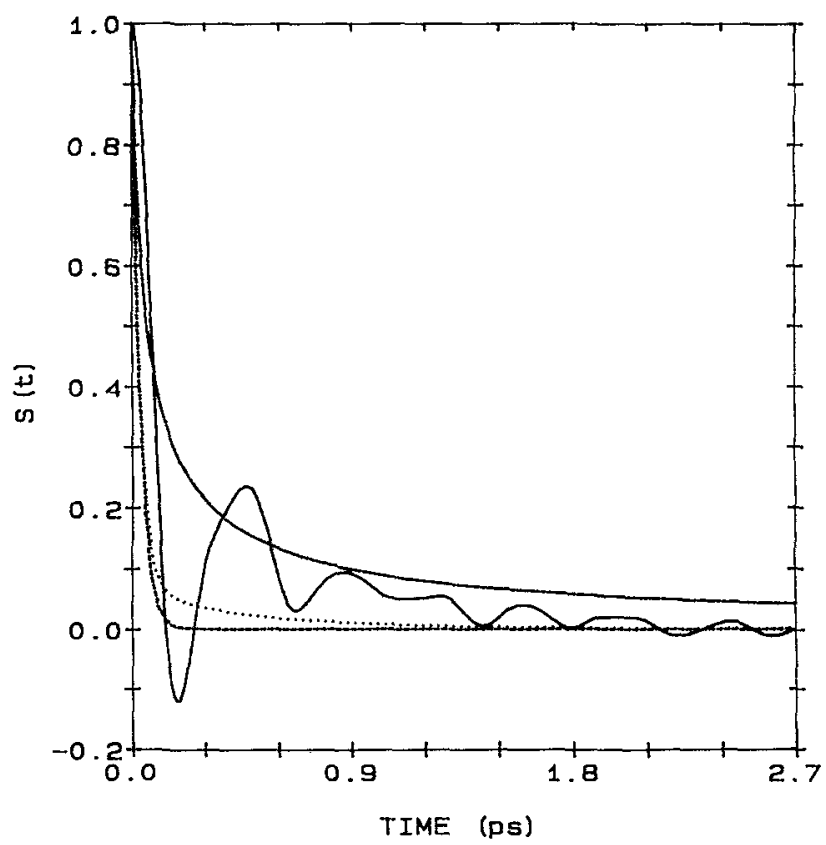

FIG. 3. Response functions $S(t)$ obtained from nonequilibrium simulation I (NESI, see the text) $(-)$, dynamical MSA $(\cdots)$, SVER, $(\cdots)$ and the continuum model (---). 
we have shown in the figure the exponential relaxation of the solvent predicted by the continuum theory.

As we observe in Fig. 3 the relaxation curve corresponding to NESI displays a fast initial decay $(\sim 0.1 \mathrm{ps})$ followed by a relatively slow decay $(\sim 2.0 \mathrm{ps})$ modulated by periodic oscillations. We also observe that after the initial response of the solvent the energy of the system is even lower than its equilibrium value [i.e., $S(t)$ becomes negative]. This may be attributed to a quick initial reorientation of dipoles towards the ion, which results in the lowering of the ion-dipole energy. But the system does not remain in this configuration with the lowest ion-dipole energy due to the presence of dipoledipole interactions. Therefore, the observed oscillations in the correlation function are the results of the timely adjustment of the dipoles to the field of the freshly created ion and the field emanating from the neighboring dipoles.

Let us now compare the relaxation behavior predicted by the continuum, MSA and SVE theories with the molecular dynamics results. Perhaps we shall mention here, that our solvent is quite accurately described by the continuum Debye model. ${ }^{19}$ The simplest model for the relaxation, the model in which the solvent is assumed to be represented by a continuum, predicts an exponentially decaying relaxation with a time constant corresponding to the longitudinal relaxation time $\tau_{l}=\tau{ }_{d}^{*} \epsilon_{\infty} / \epsilon_{0}$. Substitution into this formula of the values $2.15 \mathrm{ps}, 66.1$ and 1.0 of the Debye relaxation time, static $\left(\epsilon_{0}\right)$ and optical $\left(\epsilon_{\infty}\right)$ dielectric constants, respective$1 y,{ }^{20}$ predicts in our case a relatively short relaxation time $(0.033 \mathrm{ps})$. As Rips et al. pointed out, this time provides the lower limit to the observed relaxation. ${ }^{7}$ However, it is necessary to mention here that, for most of the experimental systems, this time provides a reasonably good estimate to the relaxation time. ${ }^{3}$

A more sophisticated theory that takes the solvent structure into account is the dynamical MSA theory. ${ }^{7}$ It predicts a multiexponential relaxation behavior with relaxation times ranging between $\tau_{l}$ and $\tau_{d}$. Although the dynamical MSA theory considers the molecular character of the solvent, it does it in a very average way and as a result no oscillatory behavior of the relaxation is predicted by it. Also, as Fig. 3 shows, the initial behavior of $S(t)$ predicted by MSA is incorrect. From the MD simulations we conclude that the initial decay may be better characterized by a Gaussian function than by an exponential one. Such a Gaussian decay of the initial response has been observed in other simulation works. ${ }^{15}$ In addition we also observe from Fig. 3, that the time to approach the asymptotic limit $[S(t)=0]$ is much longer in the framework of MSA than what MD shows.

The relaxation curve obtained from the theory based on the use of $\mathrm{SVE}^{10}$ is close, in our case, to the predictions of the simple continuum model. As we can see, the curve from the molecular dynamics displays a different behavior at the initial time but its asymptotic behavior is the same as predicted by models based on SVE and continuum description. In general we can say that the molecular dynamics displays a behavior somewhere between that predicted by the continuum theory, MSA and SVE. If one describes the relaxation by a single relaxation time $\tau^{\prime}$ defined by

$$
\tau^{\prime}=\int_{0}^{\infty} S(t) d t
$$

one obtains from the molecular dynamics simulation that $\tau^{\prime}$ is around $0.2 \mathrm{ps}$. The MSA treatment predicts a value of 0.17 $\mathrm{ps}$, which is in reasonable agreement with the relaxation time obtained from the molecular dynamics. But this agreement should be considered as fortuitous, no special physical meaning should be ascribed to it.

Let us now study how the inclusion of solvent translational motion affects its relaxation dynamics. In Fig. 4(a)
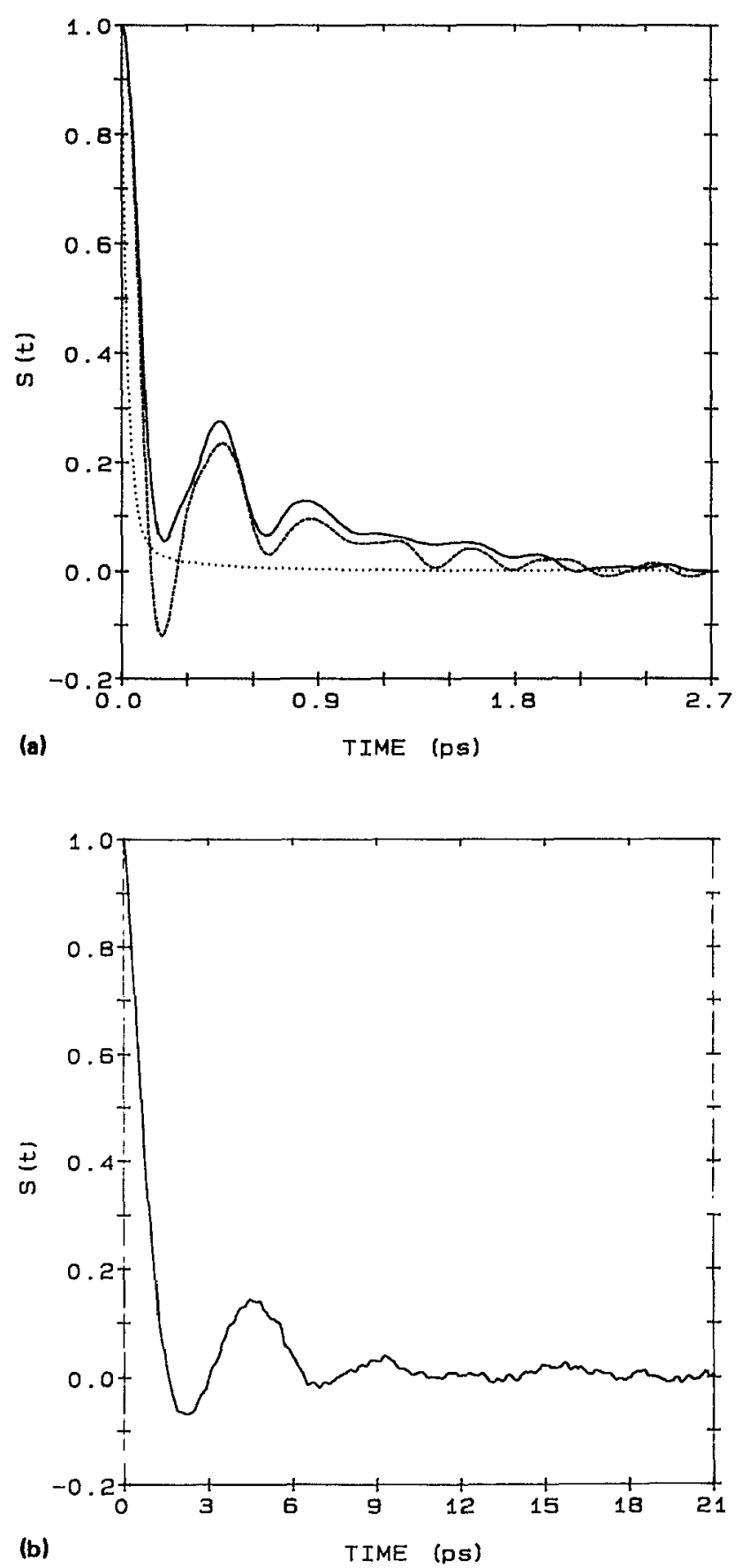

FIG. 4. (a) Response functions $S(t)$ obtained from NESI (---), NESII (-), and SVERT $(\cdots)$. (b) Response function obtained from NESIII. 
we compare the results obtained from a simulation in which the translational motion is included in the dynamics (NESII) with that of the previous simulation (NESI). Except for certain details discussed below, the two curves display similar behavior. This leads us to a conclusion that for this particular system, the rotational motion of the solvent essentially governs the overall relaxation mechanism. At least for this system, it is not surprising to observe such behavior, since it has a relatively large rotational diffusion constant $\left(D_{r}=1.167 / \mathrm{ps}\right)$ compared to its translational diffusion constant $\left(D_{t}=0.42 \AA^{2} / \mathrm{ps}\right)$.

What is the origin of the different behavior of curves NESI and NESII in Fig. 4(a), especially in the region around the first minimum? We have observed from the dynamics that the reconstruction of the solvent shells around the ion (the electrostriction effect) takes place within the first 0.2 ps at which the initial fast relaxation occurs. The reconstruction of the shell structure brings the molecules in the first two shells much closer and thus, causes the solvent molecules to feel the repulsions from their neighbors somewhat stronger than in the case when the translation is frozen. The reconstruction results in an earlier activation and stronger dipolar interactions between solvent molecules. This is especially visible from the curves in the region around $0.18 \mathrm{ps}$. However, the reconstruction does not seem to affect the long time relaxation behavior.

Figure 4(a) also includes a curve labeled SVERT. This curve was obtained from the solution of $\mathrm{SVE}^{10}$ where the effect of rotational and translational diffusion were included. Again only the long time behavior of the SVERT curve is correct, as expected. To study the relative contributions of translational or rotational diffusional modes into the total relaxation process a parameter $p^{\prime}$ defined by the equation

$$
p^{\prime}=D_{t} /\left(2 D_{r} \sigma^{2}\right)
$$

was introduced. ${ }^{10}$ Our Stockmayer solvent in which $I^{*}=0.025$ yields a value of $p^{\prime}=0.012$. As was pointed out by Bagchi and Chandra, this value of $p^{\prime}$ should not change the relative importance of translational and rotational modes. ${ }^{10}$ To study how the translational motion of the solvent influences the relaxation dynamics at larger values of $p^{\prime}$, we suppressed the fast rotations of solvent molecules by increasing their moment of inertia by a factor of 100 and performed an equilibrium (ESIII) and a series of nonequilibrium simulations (NESIII) for this new system. The equilibrium simulations show that the translational diffusion coefficient remains near that of the original system $\left(0.38 \AA^{2} / \mathrm{ps}\right)$, but the rotational diffusion coefficient decreases by a factor of 38 (to the value $0.031 / \mathrm{ps}$ ). For this new Stockmayer solvent $p^{\prime}=0.42$, and therefore we expect to see a more pronounced effect of translation on the relaxation. If we would freeze out completely the translational motion of the solvent, the dynamics in NESIII would be scaled up by a factor of 10 . Consequently, one would expect to see a complete relaxation of the solvation around the ion to take place after around 17-18 ps. However, as Fig. 4(b) indicates, a complete relaxation is achieved after around $7 \mathrm{ps}$. This points to an appreciable contribution of translational motion to the total relaxation process. Another effect that translational motion has on the relaxation is related to the Onsager snowball conjecture and is discussed below.

As we have seen from our simulation and other simulations, ${ }^{11,15}$ the relaxation of the system can be described by a fast initial decay and a subsequent slow relaxation. The existence of these two time scales are also confirmed by experiments. ${ }^{25}$ Therefore, the main questions we should ask are, what are these two (or more) time scales observed in solvation dynamics and how do they correlate with the dynamics of solvent molecules? In the previous simulations, the short time decay is assigned to the inertial motion of the solvent molecules, ${ }^{11,13,14}$ meaning that the motion of the solvent can not be described by the diffusion equation. Indeed we observe that the initial fast decay is essentially due to the immediate reaction of the solvent molecules, especially of the first solvation layer. The nature of the long time decay is clarified by Fig. 5 , where the correlation function $S(t)$ is compared to the single dipole autocorrelation function $\Phi(t)$, defined as

$$
\Phi(t)=\frac{\langle\mu(t) \cdot \mu(0)\rangle}{\left\langle\mu^{2}\right\rangle} .
$$

The autocorrelation function $\Phi(t)$ was calculated from a separate simulation performed on 512 Stockmayer particles. As we can see from Fig. 5 the long time decay of $S(t)$ is characterized by the same relaxation time as the decay of $\Phi(t)$. That does not mean that the long-time dynamics of a dipole is determined by solving the dynamical equations for a single particle, since to find a single particle correlation function in the many-body problem one has to solve the many-body problem. The agreement in long-time behavior of $S(t)$ and $\Phi(t)$ provides a reasonable conformation to the idea that the long time decay of the relaxation is due to the adjustment of the dipole orientation to the resulting field from the rest of the system.

At this stage we want to return to the discussion of the Onsager snowball effect. Onsager once commented that the relaxation of solvent around a newly created charge distribution will proceed from outside towards the solute like a snowball. ${ }^{16}$ We can check Onsager's conjecture, since molecular dynamics simulations allow us to examine the contributions to the relaxation emanating from the different solvent shells around the ion. Thus in Fig. 6(a) we show the plots of $S(t)$ vs time for each solvent shell for NESI. Initially, all the shells seem to respond similarly to the instantaneous change in the charge on the solute and this response follows a Gaussian behavior. However, the first shell molecules begin to react to the field exerted by the neighboring dipoles earlier than the molecules in other shells. Since this shell contributes more than $50 \%$ to the overall response function, one expects to observe the characteristics of the 1st shell relaxation in the overall response function. The initial relaxation behavior of the solvent molecules in the subsequent shells does not show any appreciable difference from each other, except for a small time lag in the propagation of the response. These shells are seen to be completely relaxed within 1 ps time period, but the first shell remains unrelaxed for another picosecond. The emerging picture is quite compatible with the Onsager snowball conjecture.

We represent a similar set of curves obtained from the 


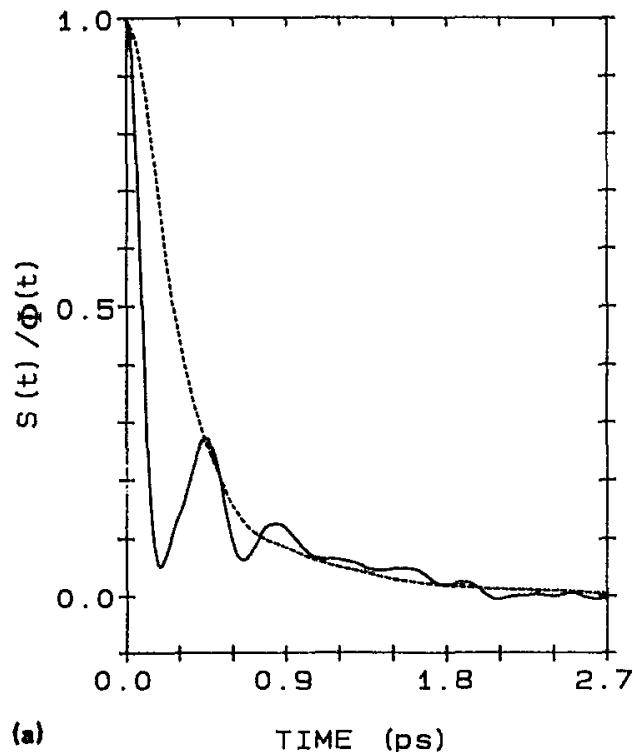

(a)

TIME (ps)

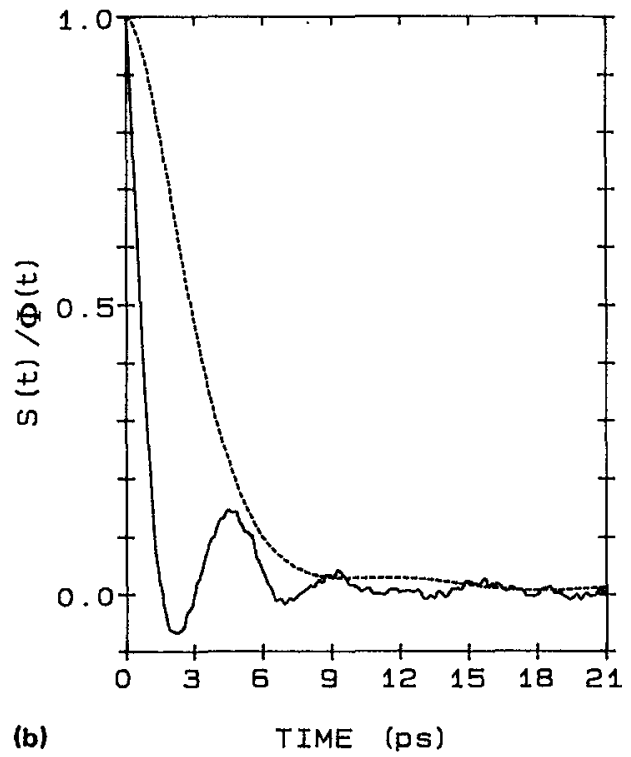

FIG. 5. (a) Response function $S(t)$ [solid line] and the single particle dipole autocorrelation function $\Phi(t)$ [dashed line]. The moment of inertia in the simulations is $I^{*}=0.025$. (b) Same as above, but $I^{*}=2.5$. trajectories from NESII in Fig. 6(b). Except for the small differences observed in the relaxation of the first shell, the curves are very similar to the ones in Fig. 6(a). Consequently, the relaxation picture presented above is also valid for the case when the translational motion of the solvent participates in the dynamics, but the rotational motion is dominant (small $p^{\prime}$ ).

In Fig. 6(c), we plot $S(t)$ of the solvent shells vs time for the case in which solvent is modified to have $I^{*}=2.5$. One can notice the difference between shells even in the behavior of the initial fast component of the relaxation. Though the decay is Gaussian, the curves have a wider spread compared to the previous cases. All the curves reach their equilibrium values at the same time (around $7 \mathrm{ps}$ ), thus displaying a breakdown of the Onsager conjecture. This agrees with the predictions of Bagchi and Chandra that pointed out that Onsager's conjecture becomes incorrect when the rotational mode is not a dominant mode in the relaxation mechanism. ${ }^{24}$

For a confirmation of our conclusions let us now turn to Figs. 7. In these we display the time dependence of the average cosine of the angle $(\theta)$ between the solvent dipole and the line joining the centers of solute and solvent. We can easily obtain the average value of $\cos (\theta)$ for a particular shell at any time $t$. Note that for a neutral solute at equilibrium with the solvent, $\langle\cos (\theta)\rangle$ is zero, since the solvent molecules are randomly oriented around the solute. However, when a charge is placed on the solute, $\langle\cos (\theta)\rangle$ may change from shell to shell and the value of $\langle\cos (\theta)\rangle$ for a particular shell depends on the distance from the ion.

Figure 7 (a) represents how the value of $\langle\cos (\theta)\rangle$ varies with time for different solvent shells when the translational motion is absent. At $t=0$, almost all the shells have their $\langle\cos (\theta)\rangle$ values close to zero showing that the initial configurations of the nonequilibrium trajectories are selected from a rather good random distribution. With the time proceeding, $\langle\cos (\theta)\rangle$ evolves towards values obtained from the equilibrium simulations.
As we see from Fig. 7(a) the molecules in the first shell reorient fast towards the ion and even "overshoot" the equilibrium angle. Subsequently, due to interactions with the neighbors and the ion the average angle oscillates on its way towards equilibrium. For the molecules in other shells the picture is similar, but due to the smaller perturbation from the ion the initial deviation in the angle is smaller; as a result the relaxation proceeds faster to the equilibrium. Not surprisingly, the values of the time taken to complete relaxation obtained from Fig. 7(a) agree quite well with those evaluated from $S(t)$ plots.

In Fig. 7(b), we present a similar set of plots for the case when the solvent translations are included in molecular dynamics (NESII). Inclusion of the translation is visible only in the features of the first shell and the final (equilibrium) values of the first three shells. The last shell displays quite the same results for the two cases. The change in the equilibrium values of the angle is due to the electrostriction, which brings shells closer to the ion and the distance from the ion determines the angle. Observation of the similar behavior of the angular relaxation confirms the rotationally dominant nature of the solvent relaxation for small value of the parameter $p^{\prime}$. Somewhat different features can be observed in the plots of $\langle\cos (\theta)\rangle$ vs time [Fig. $7(\mathrm{c})$ ] for the case in which $I^{*}=2.5$. For this case all the shells reach their equilibrium values around $7 \mathrm{ps}$ in good agreement with the results of $S(t)$ plots. Again, it proves the breakdown of the Onsager conjecture. As we see from the figure the first maximum of $\langle\cos (\theta)\rangle$ does not deviate much from its equilibrium value in this case. This is due to the competition between translational and rotational motions which contribute in this case to the relaxation.

\section{CONCLUSIONS}

We studied the dynamics of ion solvation in a simple Stockmayer fluid using molecular dynamics computer simulation technique. From our simulations we have concluded 

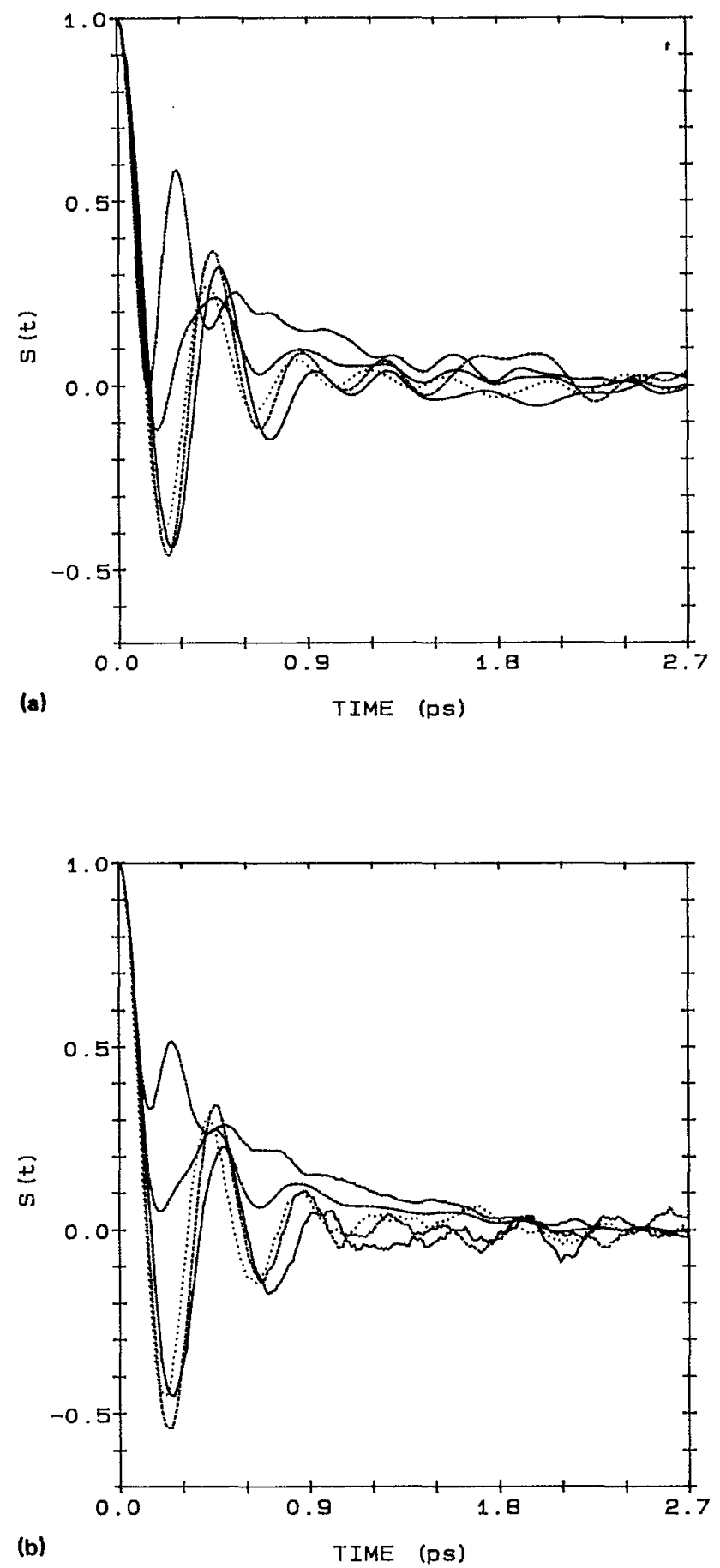

that the relaxation proceeds in two time regimes: initial short time decay regime, that can be called inertial regime, and subsequent long time decay regime, that can be called diffusional. The short time decay, as we have observed, is mostly determined by the relaxation of the solvent molecules belonging to the first solvation shell. It can be described by a correlation function, that has a Gaussian form, which is in some way a mathematical reflection of the inertial nature of the motion of the solvent molecules. We have also observed that most of the total energy has relaxed during this short time decay. The inertial character of the initial decay observed in our system may be somewhat dominant because of

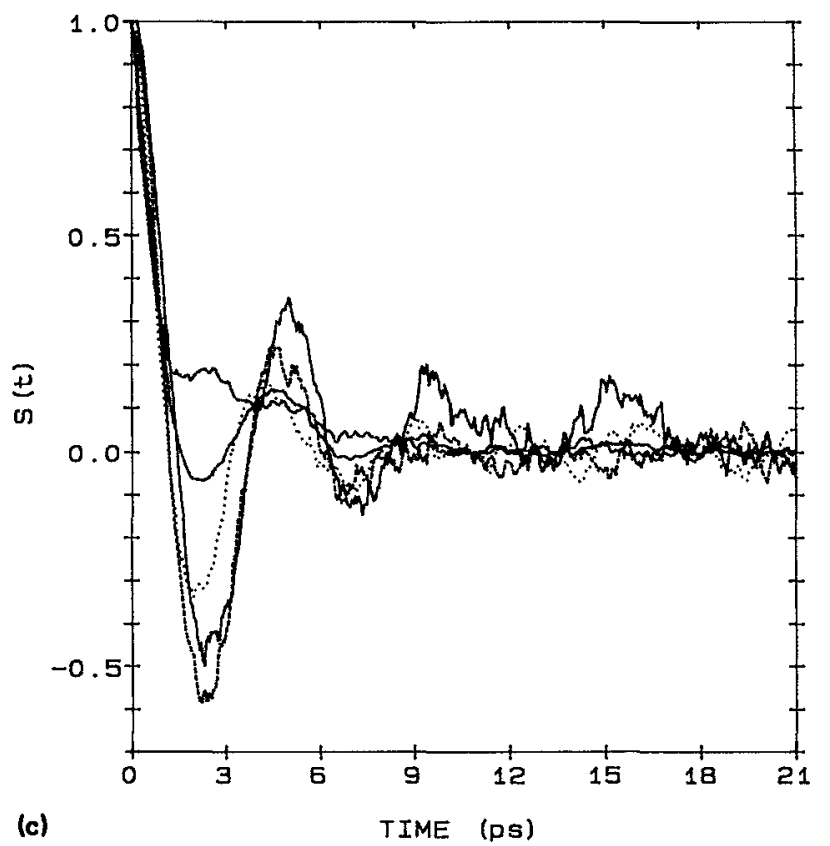

FIG. 6. Response functions (- - ) and their solvent shell components [first shell $(-\cdot)$, second shell $(\cdots)$, third shell $(--)$, and bulk $(-)]$ from (a) NESI, (b) NESII, and (c) NESIII. 

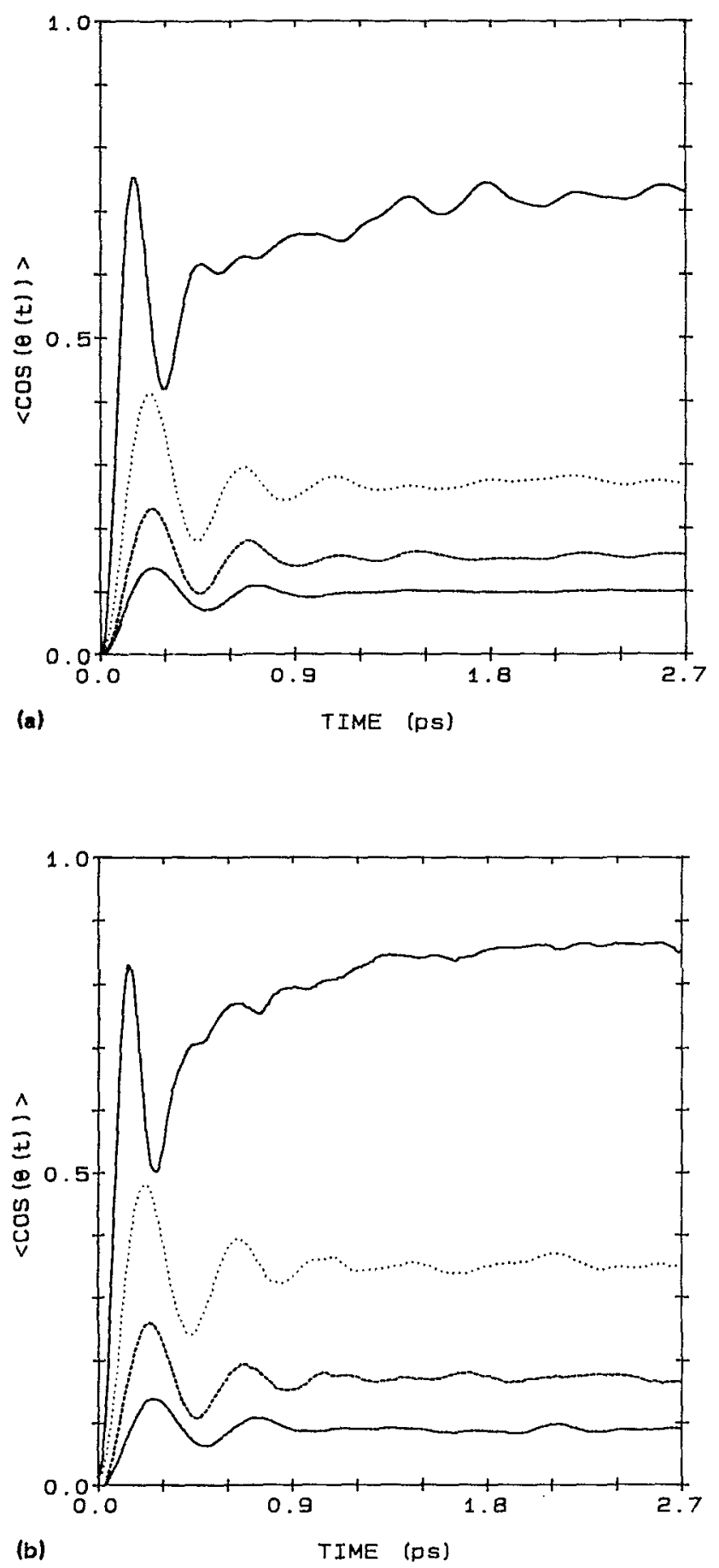

diffusional regime. Incidentally, contrary to the statements in the literature, we observe that the diffusional character of the long time decay of the solvation function $S(t)$ is not related to the diffusional rearrangement of the first solvation shell due to the translational diffusion of the solvent molecules.

We have also investigated the validity of the Onsager conjecture related to the snowball effect. We have observed that when the rotational motion is the only mode of the relaxation or when it is the dominant mode of the relaxation the Onsager conjecture holds. When the role of translational mode is increased we have observed that the Onsager conjec-

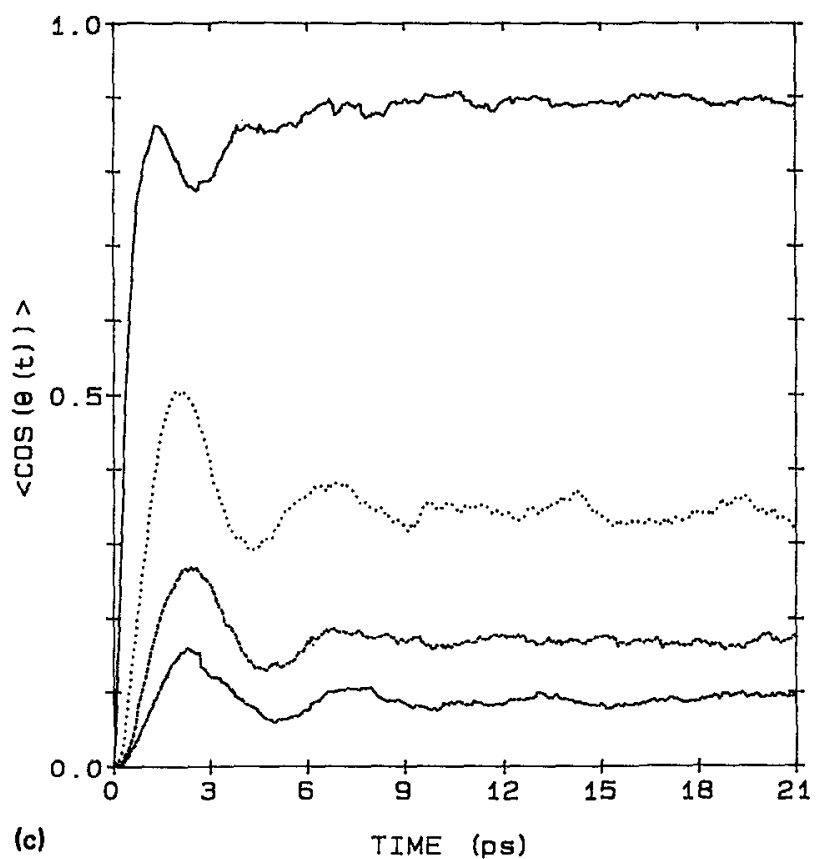

FIG. 7. Variation of $(\cos \Theta(t))$ with time for different solvation shells [first shell $(-)$, second shell $(\cdots)$, third shell $(--)$, and bulk $(\cdots)$ ] from (a) NESI, (b) NESII, and (c) NESIII.

ture is no longer valid, in total agreement with the predictions of Bagchi and Chandra. ${ }^{10,24}$

We observed that although our model fluid can be described quite well by a simple Debye-type continuum model, the relaxation process can not be described by a continuum theory. Even more sophisticated theories that we used to compare with our results from the simulations, (theories based on dynamical MSA or on SVE) are not performing that well either. Very recently Bagchi and Chandra presented a non-Markovian theory which included the viscoelastic behavior of the solvent responsible for the initial inertial response. ${ }^{26}$ Numerical studies revealed that the relaxation 
function displays short-time oscillations, followed by a slow long-time decay. ${ }^{26}$ Comparison of this new theory with the computer simulation performed on the same model will be very interesting.

Note added in proof. At the latest stage of the preparation of our manuscript we learned that simulations on similar systems were performed by Professor Nitzan and his collaborators. ${ }^{27}$

\section{ACKNOWLEDGMENTS}

This work was supported by a grant from the Office of Naval Research. The simulations were performed on the Cray YMP at the North Carolina Supercomputing Center and on the Convex C240 at UNC. Conversations with Professor J. J. Hermans, Professor B. Ladanyi, and Professor B. Bagchi were very useful.

\section{APPENDIX}

\section{The functional form of $S(t)$ in the framework of the dynamical MSA}

The dynamical MSA theory was proposed by Wolynes ${ }^{6}$ and solved exactly by Rips, Klafter, and Jortner (RKJ) ${ }^{7}$ and by Nichols and Calef. ${ }^{8}$ Here we present the final expression for the relaxation function $S(t)$ that we use in order to get the corresponding MSA curves. For the details on the development of the MSA the reader is referred to the original references. ${ }^{6-8}$

According to $\mathrm{RKJ}$ we can write an analytical expression for the Laplace transform of the relaxation function $\hat{S}(p)$

$$
\hat{S}(p)=[\hat{\chi}(p)-\hat{\chi}(0)] / p[\hat{\chi}(\infty)-\hat{\chi}(0)],
$$

where the complex admittance $\hat{\chi}(p)$ of the solvent within the MSA has the form

$\hat{\chi}(p)=\hat{\chi}_{\mathrm{MSA}}(p)=[1-1 / \hat{\epsilon}(p)] / 2 r_{i}[1+\widehat{\Delta}(p)]$.

The dynamic correction factor $\hat{\Delta}(p)$ is given by the expression

$$
\widehat{\Delta}(p)=\left(3 r_{s} / r_{i}\right)\left\{[\hat{f}(p)]^{1 / 3}+[\hat{f}(p)]^{-1 / 3}-2\right\}^{-1},
$$

where

$$
\hat{f}(p)=\hat{g}(p)-\left\{[\hat{g}(p)]^{2}-1\right\}^{1 / 2}
$$

and

$$
\hat{g}(p)=1+54[\hat{\epsilon}(p)]^{1 / 2} .
$$

$r_{i}$ and $r_{s}$ are the solute and solvent radii, respectively. In Eqs. (A2) and (A5), the dielectric susceptibility of the medium has the Debye form

$$
\hat{\epsilon}(p)=\epsilon_{\infty}+\left(\epsilon_{0}-\epsilon_{\infty}\right) /\left(1+p \tau_{d}\right) .
$$

Substitution of the Eqs. (A2)-(A6) into Eq. (A1) results in the functional form for the relaxation function $S(p)$. To get the desired function $S(t)$ the inverse Laplace transform was performed with the help of numerical techniques. ${ }^{28}$

\section{The functional form of $S(t)$ in the framework of SVE}

The Smoluchowski-Vlasov equation (SVE) was originally proposed by Wolynes and Calef ${ }^{9}$ to describe a structural relaxation of the polarization through diffusion in the mean field. Subsequently, Nicols and Calef ${ }^{8}$ solved it for the case when only rotational diffusion of the molecules was considered. Bagchi and Chandra ${ }^{10(b)}$ extended the solution to include the translational diffusion of the molecules.

The functional form of $S(t)$ in the framework of SVE is given by Eq. (19) from Ref. 10(b), which is

$$
\begin{aligned}
S(t)= & 2 \frac{r_{i}}{\pi} \int_{0}^{\infty} d k \exp \left[-t / \tau_{L}(k)\right] \\
& \times\left[\int_{k r_{i}}^{\infty} d x \frac{\sin (x)}{x}\right]^{2},
\end{aligned}
$$

where $\tau_{L}(k)$ is given by the following expression:

$$
\begin{aligned}
1 / \tau_{L}(k)= & 2 D_{R}\left\{1+p^{\prime}(k \sigma)^{2}-1 / 3 \rho\left[1+p^{\prime}(k \sigma)^{2}\right]\right. \\
& \left.\times\left(C_{\Delta}+2 C_{D}\right)\right\} .
\end{aligned}
$$

$\rho$ is the density of the solvent, $D_{R}$ is the solvent rotational diffusion coefficient, $\sigma$ is the solvent diameter, and $p^{\prime}$ is the dimensionless solvent parameter defined in Eq. (7). In Eq. (A8) $C_{\Delta}$ and $C_{D}$ are components of the direct correlation function $C(k)$. In linear theories $C(k)$ can be separated into two parts, i.e.,

$$
C(k)=C_{\Delta} \mathbf{I}+C_{D} \mathbf{D}
$$

where

$$
\mathrm{D}=3 k k-\mathbf{I} \text {. }
$$

If MSA is used to find $C(k)$, the analytical expressions for $C_{\triangle}$ and $C_{D}$ exist, and for the linear combination that appears in Eq. (A9) we get

$$
C_{\Delta}+2 C_{D}=6 K C_{\mathrm{PY}}(k ; 2 K \rho),
$$

where $C_{\mathrm{PY}}(k ; 2 K \rho)$ is the Percus-Yevick direct correlation function for hard spheres at density $2 K \rho$. $K$ is a parameter obtained from the relationships

$$
K=\xi / \eta
$$

and

$$
\eta=\pi \rho \sigma^{3} / 6
$$

where $\xi$ is the solution of the equation

$$
\frac{(1+4 \xi)^{2}(1+\xi)^{4}}{(1-2 \xi)^{6}}=\epsilon_{0} .
$$

The solution of this equation and the form of $C_{\mathrm{PY}}(k ; 2 K \rho)$ are given in Refs. 8 and 29. Note that in our numerical work we used $r_{i}=r_{s}=\sigma / 2$.

\footnotetext{
' For recent reviews and references therein see, for example, P. F. Barbara and W. Jarzeba, Adv. Photochem. 15, 1 (1990); M. Maroncelli, J. MacInnis, and G. R. Fleming, Science, 243, 1674 (1989); B. Bagchi, Annu. Rev. Phys. Chem. 40, 115 (1989); B. Bagchi, and A. Chandra, Adv. Chem. Phys. 80, 1 (1991); J. D. Simon, Acc. Chem. Res. 21, 128 (1988).

${ }^{2}$ M. Maroncelli and G. R. Fleming, J. Chem. Phys. 86,6221 (1987); E. W. Castner, Jr., M. Maroncelli, and G. R. Fleming, ibid. 86, 1090 (1987).

${ }^{3}$ W. Jarzeba, G. C. Walker, A. E. Johnson, M. A. Kahlow, and P. F. Barbara, J. Phys. Chem. 92, 7039 (1988); M. A. Kahlow, W. Jarzeba, T. J. Kang, and P. F. Barbara, J. Chem Phys. 90, 151 (1989).

${ }^{4}$ C. J. F. Bottcher and P. Bordewijk, Theory of Electric Polarization. Vol. 2 (Elsevier, Amsterdam, 1978).

${ }^{5}$ G. van der Zwan and J. T. Hynes, J. Phys. Chem. 89, 4181 (1985).
} 
${ }^{6}$ P. G. Wolynes, J. Chem. Phys. 86, 5133 (1987).

${ }^{7}$ I. Rips, J. Klafter, and J. Jortner, J. Chem. Phys. 88, 3246 (1988); 89, 4288 ( 1988 ).

A. L. Nichols III and D. F. Calef, J. Chem. Phys. 89, 3783 (1989).

${ }^{9}$ D. F. Calef and P. G. Wolynes, J. Chem. Phys. 78, 4145 (1983).

${ }^{10}$ (a) A. Chandra and B. Bagchi, Chem. Phys. Lett. 151, 47 (1988); (b) 155, 533 (1989).

"M. Maroncelli and G. R. Fleming, J. Chem. Phys. 89, 5044 (1988).

${ }^{12}$ O. A. Karim, A. D. J. Haymet, M. J. Banet, and J. D. Simon, J. Chem. Phys. 92, 3391 (1988).

${ }^{13}$ T. Fonseca and B. M. Ladanyi, J. Phys. Chem. 95, 2116 (1991).

${ }^{14}$ M. Maroncelli, J. Chem. Phys. 94, 2084 (1991).

${ }^{15}$ E. A. Carter and J. T. Hynes, J. Chem. Phys. 94, 5961 (1991).

${ }^{16}$ L. Onsager, Can. J. Chem. 55, 1819 (1977).

${ }^{17}$ F. O. Raineri, Y. Zhou, H. L. Friedman, and G. Stell, Chem. Phys. 152, 201 (1991).

${ }^{18}$ W. H. Stockmayer, J. Chem. Phys. 9, 398, 863 (1941).
${ }^{19}$ M. Neumann, Mol. Phys. 50, 841 (1983).

${ }^{20}$ M. Neumann, O. Steinhauser, and G. S. Pawley, Mol. Phys. 52, 97 (1984).

${ }^{21}$ E. L. Pollock and B. J. Alder, Physica A 102, 1 (1980); Phys. Rev. Lett. 46, 950 (1981).

${ }^{22}$ J. P. Ryckaert, G. Ciccotti, and H. J. Berendsen, J. Comp. Phys. 23, 327 (1977).

${ }^{23}$ S. H. Lee, J. C. Rasaiah, and J. B. Hubbard, J. Chem. Phys. 85, 5232 (1986); 86, 2383 (1987).

${ }^{24}$ A. Chandra and B. Bagchi, J. Chem. Phys. 91, 2594 (1989).

${ }^{25}$ S. J. Rosenthal, X. Xie, M. Du, and G. R. Fleming, J. Chem. Phys. 95 , 4715 (1991).

${ }^{26}$ A. Chandra, and B. Bagchi, Chem. Phys. 156, 323 (1991).

${ }^{27}$ A. Nitzan (private communication).

${ }^{28}$ User Manual, MATH/Library, Vol. II (version 2.0) 756 (1987).

${ }^{29}$ C. G. Gray, and K. E. Gubbins, Theory of Molecular Fluids. Vol. I, (Clarendon, Oxford, 1984). 